\title{
Reducción de la fecundidad y envejecimiento de la población de mujeres chilenas en edad fértil: 1990-2004
}

\author{
Enrique Donoso $\mathbf{S}^{1}$, Jorge Carvajal $\mathbf{C}^{1}$, \\ María Angélica D omínguez de $\mathrm{L}^{2 \mathrm{a}}$. \\ Fecundity reduction and aging \\ in fertile women population \\ in the period 1990-2004 in Chile
}

Background: Chile is in the advanced stage of demographic transition with a low natural growth and a progressively aging population. Aim: To analyze births by range of maternal age and to establish if population aging is expressed in the women's fertile age range. Material and methods: Trend analysis of births and age-specific birth rates in the period 1990-2004, in the Chilean women population of reproductive age. Raw data were obtained from the Vital Statistics Yearbooks of the National Institute of Statistics. Trends were studied by linear regression and Generalized Estimating Equation (GEE). Results: The general trend of births descended significantly from 1999 to 2004 ( $\mathrm{r}$ : -0.996; p <0.001). Births significantly decreased in the range of ages from 20 to 34 years. There was an increase in the range of ages from 35 to 44 and from 10 to 14 years. The age-specific birth rate declined in all ranges, with the exception of women aged 10 to 14 years. The number of women increased significantly at all age ranges, except for the 20-24 year-old range where no significant change was observed and the 25-29 year-old range that experienced a significant reduction. Conclusions: In the period from 1990 to 2004, there was a significant reduction of births and age-specific birth rates in Chile. There was also an increased birth rate among women aged 35 to 44 years and an aging of the fertile women population (Rev Méd Chile 2009; 137: 766-73).

(Key words: Aging; Birth rate; Fertility)

Recibido el 29 de julio, 2008. Aceptado el 7 de abril, 2009.

${ }^{1}$ Departamento de Obstetricia y Ginecología, ${ }^{2}$ Departamento de Salud Pública, Facultad de Medicina, Pontificia Universidad Católica de Chile. Santiago de Chile.

a Estadístico

$\mathrm{H}$ asta fines de la década 1950-59, la historia demográfica de Chile se caracterizaba por un crecimiento poblacional moderado a alto, debido

Correspondencia a: Dr. Enrique Donoso S. Pontificia Universidad Católica de Chile. Lira 85, 5ำ piso. Santiago, Chile. Teléfono 686-3034. E mail: edonoso@med.puc.cl a una creciente natalidad y a la reducción progresiva de la mortalidad.

Posteriormente, con la incorporación de los métodos anticonceptivos, en los inicios de la década 1960-69, comienza el descenso progresivo de la natalidad ${ }^{1}$, la reducción de las muertes maternas por aborto provocado y de las muertes 
infantiles, por control de la fecundidad en mujeres de mayor riesgo reproductivo ${ }^{1-4}$. Es así que la natalidad baja desde 274.372 nacidos vivos (nv) en 1960 a 252.157 nv en 1969; en la década siguiente continúa el descenso desde $251.231 \mathrm{nv}$ en 1970 a $234.840 \mathrm{nv}$ en 1979; en la década 198089 la natalidad aumenta desde $247.013 \mathrm{nv}$ en 1980 a 303.789 nv en 1989, registrándose en 1990 la natalidad más alta de la historia reproductiva del país con $307.522 \mathrm{nv}$; posteriormente se inicia un nuevo y sostenido descenso ${ }^{5}$.

El control poblacional de la fecundidad ha sido un tema prioritario para los países de la región, sin embargo, el diferente desarrollo económico, social y sanitario de los países de América Latina y del Caribe, hace que éstos se encuentren en distintos momentos de la transición demográfica. Chile, junto a las Antillas Holandesas, Argentina, Bahamas, Barbados, Brasil, Costa Rica, Cuba, Guadalupe, Guyana, Jamaica, Martinica, Puerto Rico, Santa Lucía, Surinam, TrinidadTobago y Uruguay, se encuentran en etapa de transición demográfica avanzada, dada por una natalidad y mortalidad moderada o baja, que se traduce en un bajo crecimiento natural de la población ${ }^{6}$.

Una de las características de la transición demográfica avanzada es que los grupos etarios que forman la población de un país no cambian simétricamente. Así los países con mayor desarrollo presentarán un aumento progresivo de los grupos etarios mayores de 65 años, determinado por una alta natalidad del pasado y una baja mortalidad actual, con un descenso de la población entre 15-64 años, debido a la reducción actual de la natalidad. Esto trae como consecuencia el envejecimiento poblacional del país y los problemas sociales, económicos y de salud pública derivados de ese fenómeno ${ }^{7}$.

Es conocido en Chile el mayor riesgo materno, perinatal e infantil asociado a las edades reproductivas extremas: menores de 20 años $^{8}$ y mayores de 40 años 9 . El mayor riesgo reproductivo de las mujeres mayores de 40 años se explica principalmente por las patologías crónicas preexistentes $^{2,9}$. Si el envejecimiento poblacional de Chile afectase al grupo de mujeres en edad reproductiva, es posible que en las próximas décadas observemos un aumento en la morbilidad y mortalidad materna y perinatal.
No se ha comunicado, específicamente, desplazamientos poblacionales en el rango de edad reproductiva, es decir un envejecimiento de la población fértil en Chile. Sin embargo, un análisis de los cambios demográficos del país, respecto de cambios en la población fértil, permitiría orientar mejor los recursos destinados al cuidado de la salud materno-perinatal.

El objetivo de este estudio es demostrar que el envejecimiento progresivo de la población, se expresa actualmente en el rango de edad fértil de la mujer, para lo que es necesario analizar según rangos de edad, la natalidad y la fecundidad en Chile en el período 1990-2004.

\section{Material y MÉTODO}

Estudio de base poblacional que analiza en la población de mujeres chilenas en edad reproductiva, la tendencia de la natalidad y de la tasa de fecundidad específica por edad, en el período comprendido entre los años 1990 y 2004.

Se define natalidad al número de nacidos vivos ocurridos en un año específico, y tasa de fecundidad específica por edad al número de nacidos vivos por mil mujeres del rango de edad respectivo, ocurridos en un año. El rango de edad estudiado comprende mujeres entre 10 y 54 años, estratificados en quinquenios y definido para este estudio como rango de edad fértil. Los datos crudos de edad materna, nacidos vivos y población estimada de mujeres se extraen de los Anuarios de Estadísticas Vitales correspondientes al período estudiado y publicados por el Instituto Nacional de Estadísticas de Chile ${ }^{10}$.

Para los cálculos de las tasas de fecundidad específicas por edad se utilizó el número de nacidos vivos ocurridos e inscritos al 31 de diciembre del año respectivo y el número de mujeres estimadas al 30 de junio de cada año.

Debido a que una misma mujer puede ser considerada más de una vez durante el período en estudio, se exploró y comprobó la existencia de autocorrelación de los datos mediante el estadígrafo de Durbin-Watson ${ }^{11}$. Razón por la cual, para el análisis de tendencia de la tasa de fecundidad específica por edad, se utilizaron modelos GEE (Generalized Estimating Equations) $^{12}$, asumiendo correlación de tipo autorregresiva (AR), en que la 
observación actual depende de la/s anterior/es. El estudio de tendencia para natalidad y población estimada de mujeres se efectuó mediante análisis de regresión lineal.

Se calculó el porcentaje de cambio de la natalidad, de la tasa de fecundidad específica por edad y de la población estimada de mujeres entre los años extremos del período (1990-2004).

Para los cálculos se utilizaron los programas estadísticos SPSS 16 y R 2.7.2. Se estableció como diferencia significativa un valor $\mathrm{p}<0,05$.

\section{Resultados}

En el período 1990-2004 se registró en Chile el nacimiento de 3.907.868 nacidos vivos. La natalidad general presentó una tendencia descendente y significativa desde 292.510 nv ocurridos e inscritos en 1990 a 230.606 nv en 2004 (R: -0,996; p <0,001), reducción equivalente a 21,2\% (Figura 1 ).

En la Tabla 1, se presenta el análisis de tendencia de la natalidad según rangos de edad materna. Hubo una reducción significativa de los nacimientos en los rangos comprendidos entre 20 y 34 años; mientras que hubo un aumento significativo en los rangos comprendidos entre 35 y 44 años y en el rango 10-14 años. No hubo cambios significativos en los rangos 15-19 años y 45-54 años (Figura 2).

En la Tabla 2, se presenta el análisis de tendencia de la población estimada de mujeres según rangos de edad. Se observa un aumento significativo del número de mujeres en todos los rangos de edad, con excepción del grupo de 20-24 años que no presentó cambios significativos, y del grupo 25-29 años que se redujo significativamente. El mayor aumento poblacional se presenta a partir de las mujeres de 35 o más años (Figura 2).

En la Tabla 3, se presenta el análisis de tendencia de la tasa de fecundidad específica según rangos de edad materna. Se observa que la tendencia de la tasa de fecundidad específica por edad fue descendente y significativa en todos los rangos de edad, con excepción del rango de mujeres de 10-14 años que no presentó cambios significativos (Figuras 2 y 3 ).

\section{DisCUSIÓN}

Este estudio confirmó que en Chile en el período 1990-2004, hubo una reducción significativa de la natalidad general y de la tasa de fecundidad específica por edad. Además, se verificó nuestra hipótesis al comprobar un envejecimiento poblacional en el rango de edad fértil de la mujer.

Es importante destacar que este estudio se inicia en 1990, año que registra la natalidad más alta de la historia reproductiva del país (307.522 nv corregidos), posteriormente se inicia un sostenido y significativo descenso equivalente a 21,2\%. Este cambio demográfico es producto de las políticas de salud reproductiva del Ministerio de

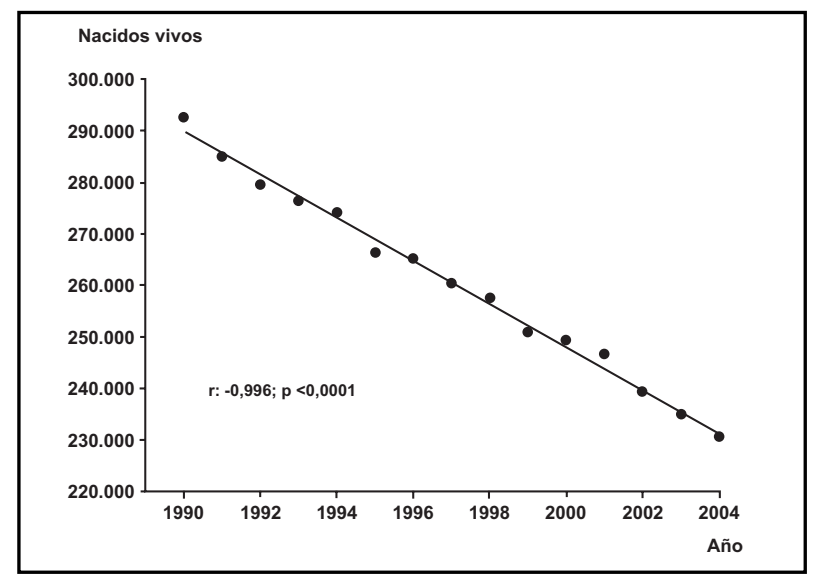

Figura 1. Tendencia de la natalidad en Chile y regresión ajustada, período 1990-2004. 
Tabla 1. Análisis de tendencia de la natalidad, según rangos de edad materna. C hile 1990-2004

\begin{tabular}{|lrrrrrr|}
\hline $\begin{array}{l}\text { Edad } \\
\text { (años) }\end{array}$ & NV $\mathbf{1 9 9 0}$ & N V $\mathbf{2 0 0 4}$ & $\begin{array}{r}\text { \% de cambio } \\
(\mathbf{1 9 9 0 - 2 0 0 4 )}\end{array}$ & $\mathbf{r}$ & Pendiente \pm EE & Valor $\mathbf{p}$ \\
\hline $10-14$ & 742 & 905 & 22,0 & 0,626 & $22,5 \pm 7,7$ & $<0,01$ \\
$15-19$ & 39.543 & 33.507 & $-15,3$ & $-0,499$ & $-220,6 \pm 106,2$ & 0,058 \\
$20-24$ & 85.292 & 53.512 & $-37,3$ & $-0,985$ & $-2.269,5 \pm 108,6$ & $<0,001$ \\
$25-29$ & 84.336 & 54.126 & $-35,8$ & $-0,984$ & $-2.012,0 \pm 99,6$ & $<0,001$ \\
$30-34$ & 52.942 & 50.571 & $-4,5$ & $-0,857$ & $-327,7 \pm 54,7$ & $<0,001$ \\
$35-39$ & 23.518 & 29.108 & 23,8 & 0,960 & $450,5 \pm 36,5$ & $<0,001$ \\
$40-44$ & 5.409 & 8.207 & 51,7 & 0,976 & $196,2 \pm 12,2$ & $<0,001$ \\
$45-54$ & 346 & 335 & $-3,2$ & $-0,254$ & $-2,2 \pm 2,3$ & 0,362 \\
\hline
\end{tabular}

NV: nacidos vivos. r: coeficiente de correlación. EE: error estándar.

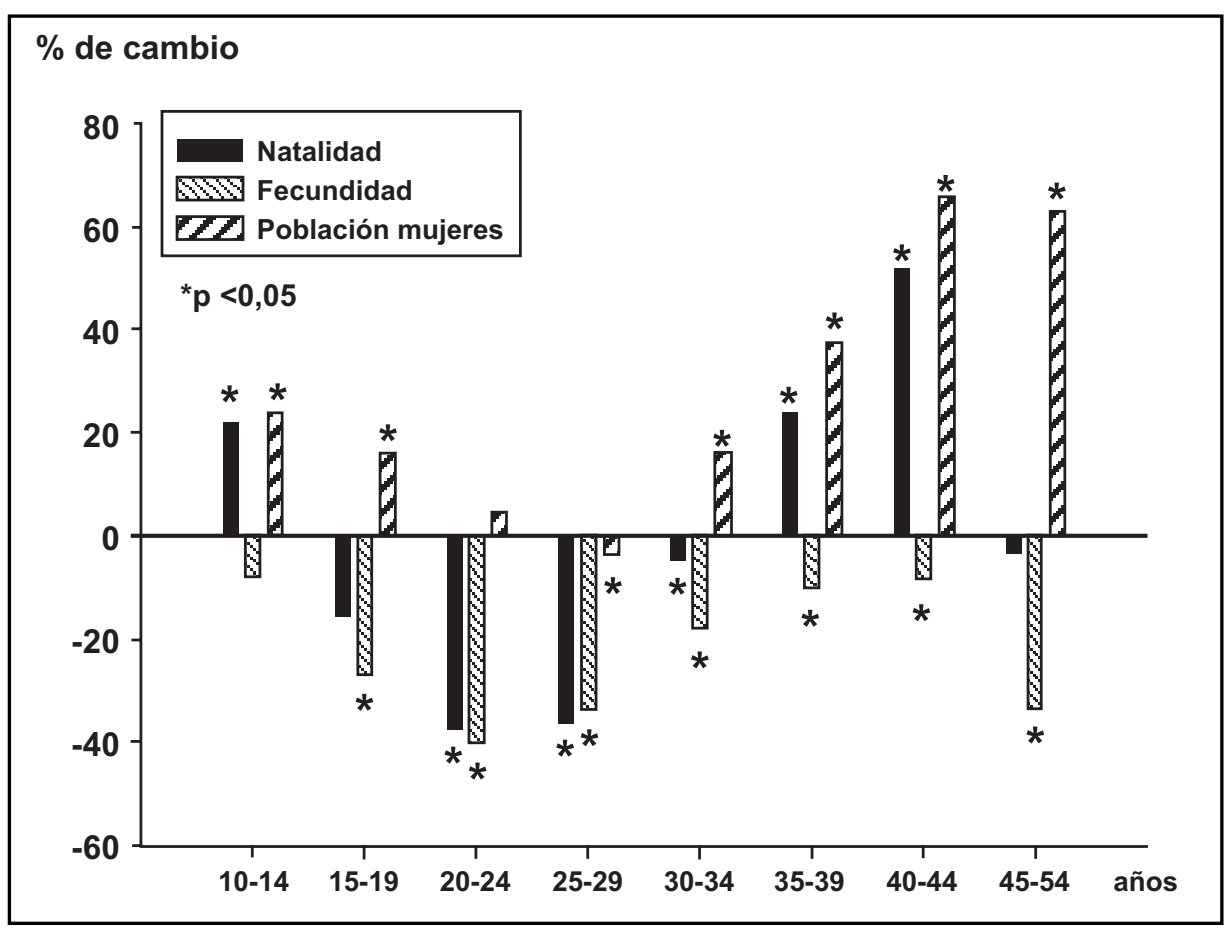

Figura 2. Porcentaje de cambio en la natalidad, tasa de fecundidad y población estimada de mujeres, entre los años 1990 y 2004.

Salud de Chile, por el aumento de la cobertura anticoncepcional ${ }^{13}$, por liberalizar la esterilización femenina y masculina desde $2000^{14}$, como también por una mayor responsabilidad de las jóvenes en la prevención del embarazo no deseado.
En efecto, las mujeres de 20 a 34 años redujeron significativamente los nacimientos ( $\sim 4.600$ nacidos vivos menos por año), mientras que las adolescentes de 15 a 19 años, pese a presentar la misma tendencia ( 220 nacidos vivos menos por año), 
Tabla 2. Análisis de tendencia de la población estimada de mujeres, según rango de edad. C hile 1990-2004

\begin{tabular}{|lcccccr|}
\hline $\begin{array}{l}\text { Edad } \\
\text { (años) }\end{array}$ & PEM 1990 & PEM 2004 & $\begin{array}{c}\text { \% de cambio } \\
(\mathbf{1 9 9 0 - 2 0 0 4 )})\end{array}$ & $\mathbf{r}$ & Pendiente \pm EE & Valor $\mathbf{p}$ \\
\hline $10-14$ & 589.180 & 728.839 & 23,7 & 0,99 & $9.836 \pm 391$ & $<0,001$ \\
$15-19$ & 609.389 & 705.903 & 15,8 & 0,856 & $6.247 \pm 1.048$ & $<0,001$ \\
$20-24$ & 611.588 & 637.470 & 4,2 & 0,042 & $102 \pm 667$ & 0,881 \\
$25-29$ & 609.421 & 588.008 & $-3,5$ & $-0,929$ & $-1.770 \pm 195$ & $<0,001$ \\
$30-34$ & 532.427 & 618.677 & 16,2 & 0,771 & $4.390 \pm 1.006$ & $<0,001$ \\
$35-39$ & 454.283 & 623.561 & 37,3 & 0,977 & $12.293 \pm 740$ & $<0,001$ \\
$40-44$ & 373.852 & 618.351 & 65,4 & 0,995 & $16.396 \pm 472$ & $<0,001$ \\
$45-54$ & 584.940 & 952.292 & 62,8 & 0,993 & $24.515 \pm 821$ & $<0,001$ \\
\hline
\end{tabular}

PEM: población estimada de mujeres. r: coeficiente de correlación. EE: error estándar.

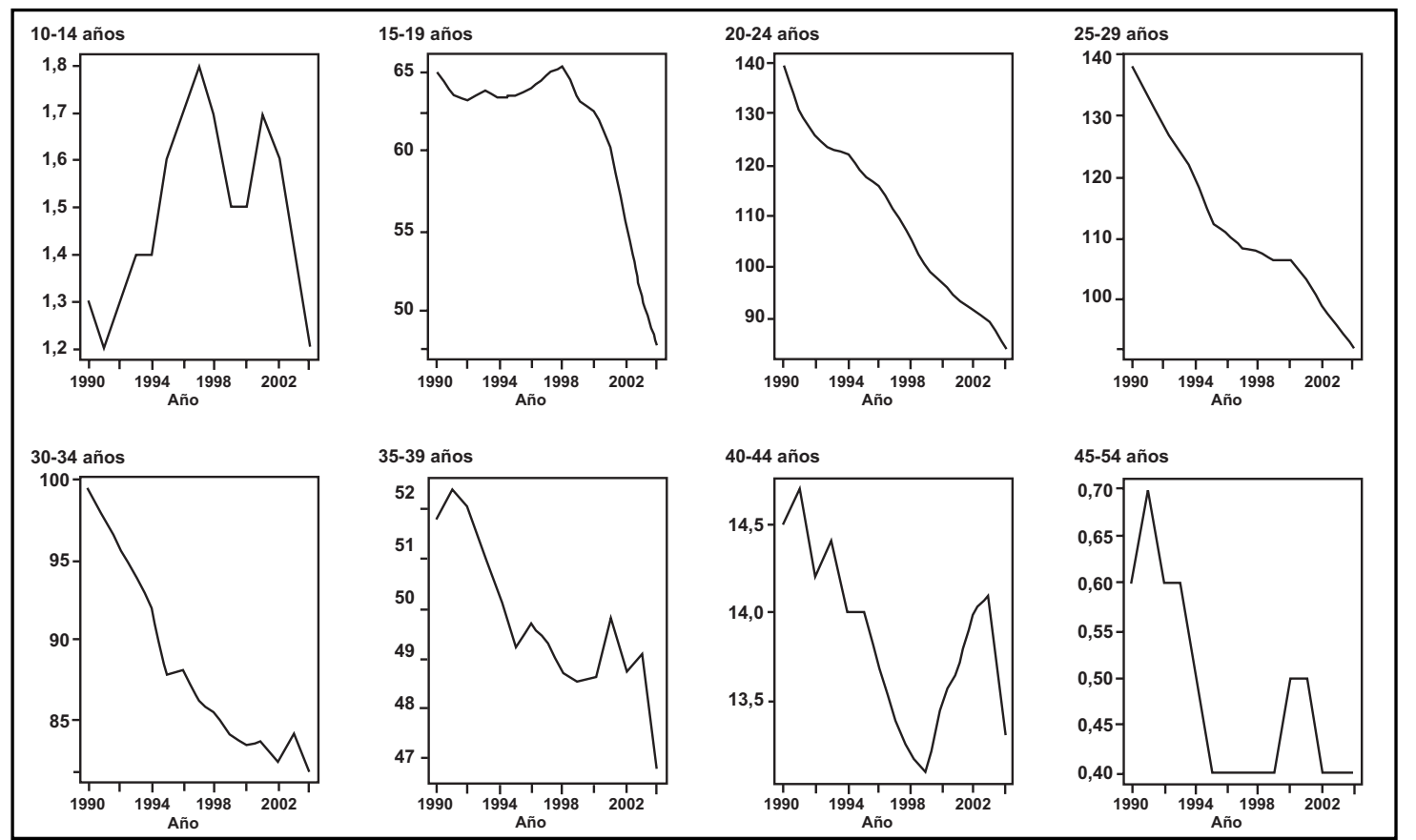

Figura 3. Tendencia de la tasa de fecundidad específica según grupo etario (nacidos vivos x 1.000 mujeres del rango de edad), período 1990-2004.

ésta no fue significativa, pero contribuyeron al descenso de la natalidad general en el período analizado.

El descenso significativo de la natalidad en el grupo de mujeres de 20 a 34 años, constituye un hecho de importancia, porque ese rango etario es considerado como "edad reproductiva ideal" o de bajo riesgo obstétrico, siendo usadas frecuentemente como grupo control en estudios de riesgo materno-perinatal ${ }^{8,9}$. Este estudio permitió detectar un aumento significativo de la natalidad en niñas de 10-14 años y en mujeres de 35 a 44 años; 


\section{Tabla 3. Análisis de tendencia de la tasa de fecundidad específica, según rangos de edad materna. Chile 1990-2004}

\begin{tabular}{|lrcccc|}
\hline $\begin{array}{l}\text { Edad } \\
\text { (años) }\end{array}$ & Tasa* 1990 & Tasa* 2004 & $\begin{array}{c}\text { \% de cambio } \\
(\mathbf{1 9 9 0 - 2 0 0 4 )}\end{array}$ & Pendiente \pm EE & Valor $\mathbf{p}$ \\
\hline $10-14$ & 1,3 & 1,2 & $-7,7$ & $-0,01 \pm 0,021$ & 0,945 \\
$15-19$ & 64,9 & 47,5 & $-26,8$ & $-1,05 \pm 0,332$ & $0,002^{* * *}$ \\
$20-24$ & 139,5 & 83,9 & $-39,9$ & $-3,77 \pm 0,154$ & $<0,001$ \\
$25-29$ & 138,4 & 92 & $-33,5$ & $-3,30 \pm 0,306$ & $<0,001$ \\
$30-34$ & 99,4 & 81,7 & $-17,8$ & $-1,26 \pm 0,202$ & $<0,001$ \\
$35-39$ & 51,8 & 46,7 & $-9,9$ & $-0,31 \pm 0,006$ & $<0,001$ \\
$40-44$ & 14,5 & 13,3 & $-8,3$ & $-0,08 \pm 0,037$ & 0,035 \\
$45-54$ & 0,6 & 0,4 & $-33,3$ & $-0,02 \pm 0,007$ & 0,021 \\
\hline
\end{tabular}

*Tasas de fecundidad específica: número de nacidos vivos x 1.000 mujeres del rango de edad. EE: error estándar. **Pendiente, EE y valor p calculados asumiendo una correlación de tipo AR(2). Para los demás casos se reportó los valores asumiendo correlación AR(1).

si consideramos que se estableció en mujeres chilenas el mayor riesgo materno-perinatal en edad reproductiva extrema $8,9,15-17$, es lógico esperar un aumento del riesgo reproductivo en la población obstétrica chilena.

El descenso de la natalidad general y el aumento de ella en edades reproductivas mayores, se explica por las mejores oportunidades que tiene la mujer chilena para su desarrollo personal y profesional en el contexto de crecientes posibilidades de educación, acceso al mundo laboral y de mejores estándares de bienestar ${ }^{18}$. Sin embargo, es importante considerar los problemas sociales y económicos, además de los biológicos ya comentados, que derivan de los cambios demográficos del país.

Un estudio socioeconómico sobre la fertilidad en Chile ${ }^{19}$, concluye que el costo de formar hijos, especialmente el educacional, es el principal determinante del descenso de la fertilidad en matrimonios o convivencias; que el mayor ingreso familiar sólo es determinante en la decisión de tener un tercer o cuarto hijo; que el mayor ingreso al campo laboral de la mujer casada o en convivencia tiende a posponer los primeros 2 hijos.

Otro estudio sobre la fertilidad en Chile ${ }^{20}$, entre otras conclusiones, muestra que la caída de la tasa de natalidad es mayor en las mujeres casadas, convergente a la tasa de las no casadas; que parte importante de la caída de la natalidad no se relaciona directamente con el mayor ingreso per cápita o con mayor participación laboral de la mujer; que la fuerte caída de la mortalidad infantil contribuye a reducir el número efectivo de hijos, puesto que es posible alcanzar el número deseado con menos nacimientos; que la caída de la fertilidad es particularmente importante en los estratos socioeconómicos más bajos, acortando la brecha con los de mayores ingresos.

Si bien la reducción de la natalidad en el período estudiado fue muy importante, ésta se inicia lenta y progresivamente desde los inicios de la década 1960-69, momento en que la tasa global de fecundidad en Chile era $\sim 5,4$ hijos por mujer ${ }^{18}$, descendiendo a 1,9 hijos por mujer en $2004^{10}$. Lo preocupante es que la baja tasa de fecundidad alcanzada, es menor que la tasa de reemplazo poblacional (2,1 hijos por mujer), lo que implica que los hijos nacidos por cada mujer no alcanzarían para renovar la población al momento del fallecimiento de sus progenitores.

Algunos países, especialmente los desarrollados, han tratado de compensar cambios similares a los observados en Chile, a través de incentivos sociales y económicos para aumentar los nacimientos e indirectamente por el potencial reproductivo de la inmigración, sin embargo, en Chile, la emigración aún supera a la inmigración ${ }^{21}$, por 
lo que será indispensable implementar incentivos a la natalidad. En este sentido consideramos un avance la reforma previsional recientemente anunciada, que establece un incentivo económico para la mujer por cada hijo nacido vivo, al cumplir 65 años $^{22}$.

El descenso significativo de las tasas de fecundidad específicas por edad en todos los grupos etarios, con excepción de las adolescentes menores de 15 años, se debe al descenso de las tasas de mortalidad general en el período estudiado ${ }^{5,10}$, que aumenta la población de mujeres, produciéndose un efecto de dilución en las tasas de fecundidad.

Es posible que la falta de cambios significativos de la tasa de fecundidad específica de las adolescentes menores de 15 años, se deba a que pese al aumento significativo de la natalidad, ésta es cuantitativamente muy baja ( 22 nacidos vivos promedio por año) comparada con el aumento de la población de mujeres de ese rango de edad (9.836 mujeres promedio por año). Además, este grupo posee una tasa de fecundidad muy baja, por la inclusión de niñas de 10 a 12 años, muchas de ellas premenárquicas, con alta frecuencia de ciclos anovulatorios o sin actividad sexual ${ }^{23}$.

De especial preocupación es el grupo de mujeres entre 45 y 54 años que presentaron el mayor crecimiento poblacional del período (aumento promedio de 24.515 mujeres por año), que aportan un promedio de $\sim 340$ nacidos vivos por año, con reducción significativa de la tasa de fecundidad específica por edad, por efecto de dilución por el aumento significativo de mujeres de ese rango etario, como también por incluir mujeres con alto incentivo anticoncepcional, muchas de ellas histerectomizadas o con ligadura tubaria, o en perimenopausia con reducción espontánea de su fecundidad $^{24}$, sin embargo, constituyen un grupo de muy alto riesgo materno-perinatal ${ }^{9,17}$.

El envejecimiento poblacional de las mujeres en edad fértil en el período estudiado, se debe a los cambios de la natalidad y mortalidad en el período 1936-1994, correspondiente al rango de los años de nacimiento de la población estudiada, y que comprende alrededor de 25 años antes de la incorporación poblacional de los métodos anticonceptivos en Chile ${ }^{1}$. El actual análisis comprende años con tasas de natalidad altas (1936: 34,6/ 1.000 habitantes) a moderadas (1994: 20,5/1.000 habitantes), con tasas de mortalidad altas (1936: 25,3/1.000 habitantes) a bajas (1994: 5,4/1.000 habitantes) y estables en los últimos 10 años (2004: 5,4/1.000 habitantes $)^{5,10}$. Esto trae como consecuencia que el mayor porcentaje de cambio positivo predomine en la población de mujeres mayores de 35 años, por nacer en momentos de alta natalidad y baja mortalidad actual; el resultado bruto es un envejecimiento de la población femenina en edad reproductiva.

El cambio demográfico que comunicamos en esta investigación es importante de considerar en la asignación de recursos al cuidado de la salud materno-perinatal. Si para la planificación se consideran las tasas de fecundidad específica por edad, todas las cuales descendieron, podría haber una reducción de recursos. Pero si se considera la natalidad, los recursos deberían aumentar en el grupo de alto riesgo obstétrico y readecuarse en el de bajo riesgo. Si los recursos no se asignan apropiadamente, es posible que estos cambios demográficos impidan el descenso esperado de la mortalidad materna a $2010^{13}$, ya que desde el año 2000, las enfermedades maternas concurrentes están muy cerca de ser la primera causa de muerte $^{25}$, las que se concentran especialmente en embarazadas mayores de 40 años $2,9,17$.

\section{Conclusión}

Este estudio confirma que en Chile, en el período 1990-2004, hubo una reducción significativa de la natalidad general y de las tasas de fecundidad específica por edad. Los datos que hemos presentado prueban nuestro postulado respecto del envejecimiento poblacional en el rango de edad fértil de la mujer, y sugieren que los recursos en salud materna y perinatal deben ser planificados considerando estos antecedentes. 


\section{REFERENCIAS}

1. Viel B, Campos W. Chilean history of infant and maternal mortality, 1940-1985. Perspec Int Planif Fam 1987; (Spec №): 24-8.

2. Donoso E. Reducción de la mortalidad materna en Chile de 1990 a 2000. Rev Panam Salud Pública 2004; 15: 326-30.

3. Szot J, Moreno C. Mortalidad por aborto en Chile: Análisis epidemiológico 1985-2000. Rev Chil Obstet Ginecol 2003; 68: 309-14.

4. Taucher E, Jofré I. Mortalidad infantil en Chile: el gran descenso. Rev Méd Chile 1997; 125: 1225-35.

5. Aguila A, Muñoz $H$. Tendencia de la natalidad, mortalidad general, infantil y neonatal en Chile desde el año 1850 a la fecha. Rev Méd Chile 1997; 125: $1236-45$.

6. CEPAL. Temas de Población y Desarrollo: Transición Demográfica. Disponible en: http://www.eclac.cl/ celade/noticias/noticias/2/23462/PyDTD_1.pdf. [Consultado el 26 de agosto de 2006].

7. CEPAL. América Latina y el Caribe: El envejecimiento de la población, 1950-2050. Boletín Demográfico 2003; 72:1-330. Disponible en: http://www.eclac.cl/ publicaciones/xml/1/13371/LCG2211.pdf. [Consultado el 11 de febrero de 2007].

8. Donoso E, Becker J, Villarroel L. Natalidad y riesgo reproductivo en adolescentes de Chile, 1990-1999. Rev Panam Salud Pública 2003; 14: 3-8.

9. Donoso E, Villarroel L. Edad materna avanzada y riesgo reproductivo. Rev Méd Chile 2003; 130: 55-9.

10. Instituto Nacional de Estadísticas de Chile. Anuarios de Estadísticas Vitales de Chile, 1990-2004.

11. Durbin J, Watson GS. Testing for serial correlation in least squares regression I. Biometrika 1950; 37: 409-28.

12. HedekER D, GibBons RD. Generalized Estimating Equations (GEE) Models. En: Longitudinal Data Analysis. Hedeker D, Gibbons RD (eds). Wiley Series in Probability and Statistics. Hoboken, New Jersey: John Wiley \& Sons, Inc.; 2006; 131-46.

13. MINSAL. Objetivos sanitarios para la década 20002010. Evaluación a mitad de período. Disponible en: http://www.minsal.cl/ici/destacados/ Evaluacion_de_los_Objetivos_Sanitarios_de_Impacto_a_mitad_de_periodo.pdf. [Consultado el 13 de septiembre de 2007].

14. MINSAL. Resolución Exenta № 2326 del 30 de noviembre de 2000. Fija directrices para los Servicios de Salud sobre esterilización femenina y mascu- lina. Disponible en: http://www.minsal.cl/juridico/ RESOLUCION_2326_00.doc. [Consultado el 2 de febrero de 2008].

15. Donoso E, Becker J, Villarroel L. Embarazo en la adolescente chilena menor de 15 años. Análisis de la última década del siglo XX. Rev Chil Obstet Ginecol 2001; 66: 391-6.

16. Donoso E, Becker J, Villarroel L. Evolución de la natalidad y del riesgo reproductivo en mujeres de 40 o más años en la década de los 90. Rev Chil Obstet Ginecol 2002; 67: 139-42.

17. Donoso E, Carvajal JA. Maternal, perinatal, and infant outcome of spontaneous pregnancy in the sixth decade of life. Maturitas 2008; 59: 381-6.

18. Instituto Nacional de Estadísticas de Chile. Fecundidad en Chile. Disponible en: http://www.ine.cl/ canales/sala_prensa/archivo_documentos/enfoques/2006/pdf/fecundidad1.pdf. [Consultado el 10 de febrero de 2007].

19. Larrañaga O. Fertilidad en Chile, 1960-2003. Disponible en: http://sechi.facea.uchile.cl/sechi/Invitados_2/larranaga_osva.pdf. [Consultado el 11 de febrero de 2007].

20. CERDA RA. ¿Dónde están los niños? Determinantes socioeconómicos e institucionales de la disminución de la tasa de fertilidad en Chile. Disponible en: http://sechi.facea.uchile.cl/sechi/invitados_1/ cerda_rcer.pdf. [Consultado el 11 de febrero de 2007].

21. CEPAL. Migración Internacional. Observatorio Demográfico 2006; 1: 1-205. Disponible en: http:// www.cepal.org/publicaciones/xml/8/27498/ Observatoriodemografico.pdf. [Consultado el 11 de febrero de 2007].

22. Gobierno de Chile, Ministerio del Trabajo y Previsión Social. Reforma Previsional. Disponible en: http:// www.reformaprevisional.cl/reforma_previsional/inicio/beneficio_3.htm. [Consultado el 13 de julio de 2008].

23. Molina R, Molina T, González E. Madres niñasadolescentes de 14 años y menos. Un problema de salud pública no resuelto en Chile. Rev Méd Chile 2007; 135: 79-86.

24. Quevedo I, Flores M, Castillo M. Características reproductivas de las mujeres consultantes en servicios de urgencia general de la VIII Región. Rev Méd Chile 2005; 133: 929-34.

25. Donoso E. Mortalidad materna en Chile, 2000-2004. Rev Chil Obstet Ginecol 2006; 71: 246-51. 